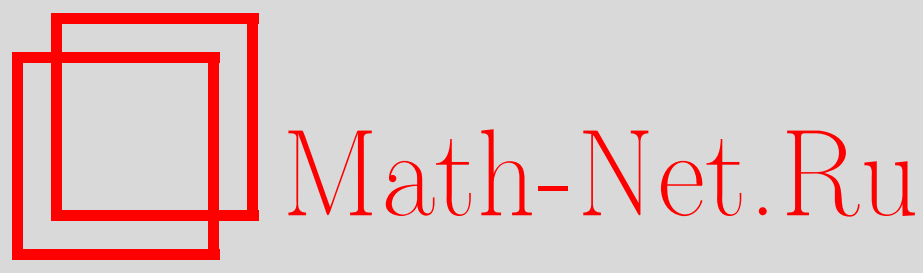

О. В. Грошев, О существовании и единственности классических решений задачи Коши на неглобально гиперболических многообразиях, ТМФ, 2010, том 164, номep 3, 441-446

DOI: https://doi.org/10.4213/tmf6555

Использование Общероссийского математического портала Math-Net.Ru подразумевает, что вы прочитали и согласны с пользовательским соглашением http://www . mathnet.ru/rus/agreement

Параметры загрузки:

IP : 52.205 .19 .152

26 апреля 2023 г., 16:57:22

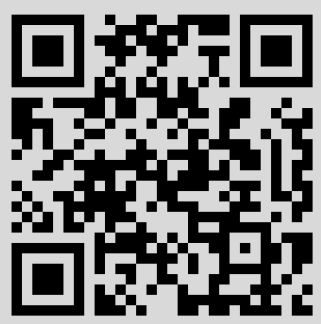




\title{
О СУЩЕСТВОВАНИИ И ЕДИНСТВЕННОСТИ КЛАССИЧЕСКИХ РЕШЕНИЙ ЗАДАЧИ КОШИ НА НЕГЛОБАЛЬНО ГИПЕРБОЛИЧЕСКИХ МНОГООБРАЗИЯХ
}

\begin{abstract}
Задача Коши для волнового уравнения рассматривается на пространстве Мизнера - неглобально гиперболическом многообразии, содержащем замкнутые времениподобные кривые. Доказывается, что существование и единственность классического решения эквивалентны условиям самосогласованности гораздо более строгим, чем конечный набор точечных условий, возникающих для этой задачи на плоскости Минковского с присоединенной ручкой.
\end{abstract}

Ключевые слова: неглобально гиперболические многообразия, волновое уравнение, задача Коши.

\section{1. ВВЕДЕНИЕ}

В настоящее время существует хорошо развитая теория гиперболических уравнений на глобально гиперболических многообразиях [1], [2]. Глобально гиперболическое многообразие - это ориентируемое по времени лоренцево многообразие $(M, g)$, которое не имеет замкнутых времениподобных кривых, и множество времениподобных путей между любыми двумя точками которого компактно. В работе [3] доказано, что все глобально гиперболические многообразия диффеоморфны $\mathbb{R}^{1} \times \Sigma$, где $\Sigma$ - поверхность Коши; таким образом, все они имеют тривиальную топологию.

Вместе с тем большое число неглобально гиперболических многообразий предоставляет общая теория относительности. Эти многообразия являются решениями полевых уравнений Эйнштейна; наиболее известными примерами являются пространства анти-де Ситтера, Геделя и Готта, машина времени Дойча-Полицера и многие другие [4]-[6]. Все эти пространства имеют замкнутые времениподобные кривые, которые с физической точки зрения означают возможность путешествия во времени.

* Математический институт им. В. А. Стеклова РАН, Москва, Россия. E-mail: groshev@mi.ras.ru 
Интересным является вопрос построения теории поля на подобных пространствах, в частности вопрос о том, на каких пространствах разрешимо волновое уравнение

$$
\square u=\frac{1}{\sqrt{-g}} \partial_{\alpha}\left(g^{\alpha \beta} \sqrt{-g} \partial_{\beta} u\right)=0 .
$$

Из работ Лерэ известно, что на глобально гиперболических многообразиях задача Коши для волнового уравнения разрешима всегда.

Вопрос существования классических решений задачи Коши на неглобально гиперболических многообразиях рассматривался в работах [7]-[10]. В частности, в последней работе доказаны существование и единственность классического решения уравнения Клейна-Гордона на факторе пространства анти-де Ситтера, а также на кротовых норах специального класса.

В работе [11] было доказано, что на плоскости Минковского с присоединенной ручкой (модифицированная машина Дойча-Полицера) классическое решение существует и единственно при выполнении конечного набора точечных условий самосогласованности; кроме того, в ней были приведены примеры, когда полученное решение путешествует во времени, а также затронут вопрос существования обобщенных решений. В работах [12], [13] было доказано, что машину Дойча-Полицера нельзя вложить в асимптотически плоское лоренцево многообразие так, чтобы метрика осталась несингулярной. Эти доказательства остаются верными и для модифицированного пространства.

В настоящей работе мы сравниваем результаты статьи [11] с результатами для волнового уравнения на пространстве Мизнера. Мы доказываем, что существование классического решения на нем эквивалентно таким условиям для начальных данных, которые в точности запрещают путешествия во времени. Отметим, что наша работа также мотивирована изучением возможности создания кротовых нор и неглобально гиперболических регионов при столкновении частиц на высоких энергиях [14].

\section{2. РЕЗУЛЬТАТЫ ДЛЯ ПЛОСКОСТИ С РУЧКОЙ}

Напомним основные результаты, полученные для плоскости с ручкой. Конструкция плоскости с ручкой следующая.

На полуплоскости $\mathbb{R}_{+}^{2}=\left\{(x, t) \in \mathbb{R}^{2} \mid t>0\right\}$ рассмотрим два вертикальных интервала $S_{1}$ и $S_{2}$ длины $\ell>0$ :

$$
S_{i}=\left\{(x, t) \in \mathbb{R}_{+}^{2} \mid x=x_{i}, t_{i}<t<t_{i}+\ell\right\} .
$$

Предположим, что

$$
0<x_{2}-x_{1}<t_{2}-t_{1}+\ell ;
$$

таким образом, вектор $I=\left(x_{2}-x_{1}, t_{2}-t_{1}\right)$, переводящий $S_{1}$ в $S_{2}$, времениподобен.

Склеим стороны отрезков, как показано на рис. 1, а именно склеим "внутренние" стороны разрезов друг с другом и аналогично "внешние" стороны друг с другом. Получившееся многообразие имеет две особые точки - концы отрезков.

Любое гладкое поле, заданное на рассматриваемом многообразии, будет удовлетворять определенным условиям склейки на разрезах $S_{1}$ и $S_{2}$. Обратно, если поле 


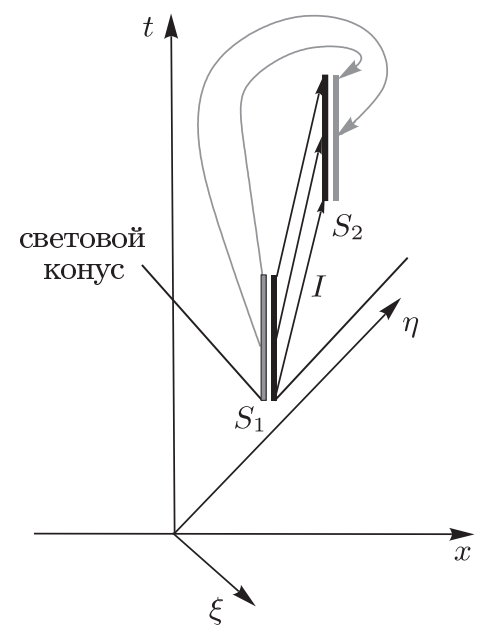

Рис. 1. Плоскость Минковского с двумя разрезами, склеенными определенным образом. Идентификация точек "внешних" и "внутренних" сторон разрезов указана линиями со стрелками.

дифференцируемо в области $\Omega=\mathbb{R}_{+}^{2} \backslash \bar{S}_{1} \cup \bar{S}_{2}$ и удовлетворяет этим условиям склейки, то оно гладко на рассматриваемом многообразии.

Рассмотрим волновое уравнение на этом многообразии:

$$
u_{t t}-u_{x x}=0
$$

в $\Omega$ с начальными условиями

$$
\left.u\right|_{t=0}=\varphi,\left.\quad u_{x}\right|_{t=0}=\psi,
$$

где $\varphi \in C^{2}(\mathbb{R}), \psi \in C^{1}(\mathbb{R})$. Наложим следующие условия склейки:

$$
u\left(X_{ \pm}\right)=u\left(X_{\mp}+I\right), \quad u_{x}\left(X_{ \pm}\right)=u_{x}\left(X_{\mp}+I\right),
$$

где $X=(x, t) \in S_{1}$, а

$$
u\left(X_{ \pm}\right)=\lim _{x \rightarrow x_{1} \pm 0} u(x, t) .
$$

Предполагается, что указанные пределы справа и слева существуют.

Введем следующее определение.

ОПРЕДЕЛЕНИЕ 1. Классическим решением задачи (3)-(5) называется функция $u \in C^{2}(\Omega) \cap C^{1}(\Omega \cup\{t=0\})$, удовлетворяющая условиям (3)-(5) в предположении, что указанные в условиях (5) пределы справа и слева существуют.

В дальнейшем будем пользоваться обозначениями

$$
c_{i}=x_{i}-t_{i}, \quad d_{i}=x_{i}+t_{i} ;
$$

здесь $i$ может быть равно 1 или 2 . Будем обозначать через $\Phi$ следующий (упорядоченный) набор функций:

$$
\Phi(x)=\left(\varphi^{(i)}(x), i=0,1,2 ; \quad \int_{a}^{x} \psi(s) d s ; \quad \psi^{(j)}(x), j=0,1\right) .
$$


Обозначим через $\Psi$ вектор

$$
\Psi=\left(\Phi\left(c_{1}-\ell\right), \Phi\left(c_{2}-\ell\right), \Phi\left(c_{1}\right), \Phi\left(c_{2}\right), \Phi\left(d_{1}+\ell\right), \Phi\left(d_{2}+\ell\right), \Phi\left(d_{1}\right), \Phi\left(d_{2}\right)\right) .
$$

Была доказана следующая теорема.

Теорема 1. Для существования классического решения задачи (3)-(5) необходимым и достаточным является выполнение равенства

$$
L \Psi=0,
$$

где L - некоторая специальная матрица ранга 10.

ЗАмЕчАниЕ 1. Поскольку условие (8) представляет собой конечное число условий для значений в точках, пространство функций, удовлетворяющих им, достаточно обширно. В частности, в работе [11] были построены примеры решений, которые проходят по региону с нарушенной глобальной гиперболичностью, и, таким образом, "путешествуют во времени".

\section{3. РЕЗУЛЬТАТЫ ДЛЯ ПРОСТРАНСТВА МИЗНЕРА}

Пространство Мизнера - это хорошо известное в общей теории относительности плоское двумерное пространство-время. Оно является фактором $\mathbb{R}^{1,1} /\langle\mathcal{B}\rangle$ пространства Минковского по свободной группе, порожденной бустом $\mathcal{B}$. Проще всего это многообразие представить в системе координат $(\xi, \eta)$, где

$$
\xi=-(x+t), \quad \eta=x-t .
$$

В этих координатах буст $\mathcal{B}$ задается следующим образом:

$$
\mathcal{B}:(\xi, \eta) \mapsto\left(B \xi, B^{-1} \eta\right) .
$$

Он сохраняет лоренцеву метрику $d s^{2}=-d \xi d \eta$. В качестве фундаментальной области действия $\mathcal{B}$ можно взять полосу $\left\{\xi_{0}<\xi<B \xi_{0}\right\}$. Само пространство Мизнера получается (см. рис. 2) после отождествления границ фундаментальной области с помощью соотношения

$$
\left(\xi_{0}, \eta\right) \sim\left(B \xi_{0}, B^{-1} \eta\right)
$$

На получившемся многообразии существует единственная замкнутая нулевая геодезическая $\eta=0$. Она делит пространство на два региона - "прошлое" и "будущее". В прошлом существуют глобальные поверхности Коши и не существует замкнутых времениподобных кривых, тогда как в будущем через каждую точку проходит замкнутая времениподобная кривая.

Сформулируем задачу Коши для волнового уравнения на пространстве Мизнера. Волновое уравнение $\square u=0$ в координатах $(\xi, \eta)$ имеет вид

$$
u_{\xi \eta}=0
$$

в $\Theta=\left\{\xi_{0}<\xi<B \xi_{0}, t>0\right\}$. Начальные данные мы задаем на поверхности Коши, которая лежит в прошлом и поэтому является пространственноподобной. В качестве такой поверхности можно взять $\Sigma=\{t=0\}$. Итак, задача Коши состоит в том, что у нас заданы $u$ и $\nabla u$ на $\Sigma$ :

$$
\left.u\right|_{t=0}=\varphi,\left.\quad \nabla u\right|_{t=0}=\psi .
$$




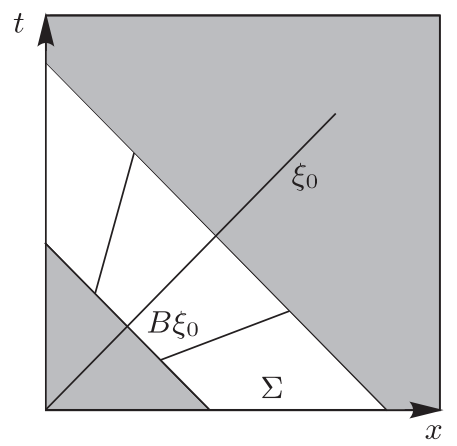

Рис. 2. Пространство Мизнера получается после отождествления границ фундаментальной области (белая). После отождествления отрезки, отмеченные на фундаментальной области, превращаются в окружности.

Кроме того, нам нужно задать условия склейки $u$ на границах фундаментальной области. Сделаем это следующим образом:

$$
\begin{aligned}
u\left(\xi_{0}, \eta\right) & =u\left(B \xi_{0}, B^{-1} \eta\right), \\
\nabla u\left(\xi_{0}, \eta\right) & =\mathcal{B} \nabla u\left(B \xi_{0}, B^{-1} \eta\right) .
\end{aligned}
$$

Нас интересуют классические решения этой задачи.

ОПРЕДЕЛЕНиЕ 2. Классическим решением задачи (9)-(12) называется функция $u \in C^{2}(\Theta) \cap C^{1}(\bar{\Theta})$, удовлетворяющая условиям (9)-(12).

Верна следующая теорема.

Теорема 2. Для существования классического решения задачи (9)-(12) необходимо и достаточно выполнение условий

$$
\varphi\left(\xi_{0}\right)=\varphi\left(B \xi_{0}\right), \quad \psi\left(\xi_{0}\right)=B \psi\left(B \xi_{0}\right), \quad \varphi^{\prime}=\psi .
$$

При этом классическое решение единственно и имеет вид волнь, идущей влево: $u(\xi, \eta)=\varphi(\xi)$.

ДокАЗАТЕЛЬСтво. Функция $u$ в области $\Theta$ представляется в виде суммы:

$$
u(\xi, \eta)=f(\xi)+g(\eta) .
$$

Запишем для нее условие (11):

$$
f\left(\xi_{0}\right)+g(\eta)=f\left(B \xi_{0}\right)+g\left(B^{-1} \eta\right), \quad \eta \in \mathbb{R}^{1} .
$$

Подставив $\eta=0$, получим

$$
f\left(\xi_{0}\right)=f\left(B \xi_{0}\right),
$$

откуда, возвращаясь к условию (15), получаем

$$
g(\eta)=g\left(B^{-1} \eta\right)
$$

Это означает, что мы можем задать функцию $g$ на полуинтервале $\left[\eta_{0}, B \eta_{0}\right)$, где $\eta_{0}>0$, и продлить ее по формуле (17) на весь луч $\eta>0$. Если $g \neq$ const, то при 
подходе к $\eta=0$ колебания функции $g$ будут учащаться, что приведет к разрыву. Следовательно, $g=$ const, а это равносильно условию $\varphi^{\prime}=\psi$.

Из этого следует, что $f=\varphi$, и условие (16) есть первое условие в (13). Подставив сумму (14) в условие склеивания градиента, получаем $f^{\prime}\left(\xi_{0}\right)=B f^{\prime}\left(B \xi_{0}\right)$. Таким образом, условия (13) являются необходимыми. Они являются достаточными, поскольку все рассуждения можно провести в обратном направлении. Единственность решения следует из того, что решение с нулевыми начальными данными будет нулевым. Теорема доказана.

ЗАмечАние 2. Качественно решения такого вида сильно отличаются от решений из раздела 2. А именно такие решения не путешествуют во времени, поскольку имеют только левую моду, которая не идет вдоль замкнутых времениподобных кривых.

Благодарности. Автор благодарен И. В. Воловичу и участникам семинара НОЦ МИАН за полезные обсуждения. Работа выполнена при поддержке программы "Ведущие научные школы” (гранты НШ-7675.2010.1 и НШ-8784.2010.1) и РФФИ (грант № 09-01-12161-офи_м).

\section{Список литературы}

[1] I. Petrowsky, Матем. сб., 2(44):5 (1937), 815-870.

[2] Ж. Лере, Гиперболические дифференииальные уравнения, Наука, М., 1984.

[3] A. N. Bernal, M. Sánchez, Comm. Math. Phys., 257:1 (2005), 43-50.

[4] С. Хокинг, Дж. Эллис, Крупномасштабная структура пространства-времени, Мир, M., 1977.

[5] H. D. Politzer, Phys. Rev. D, 49:8 (1994), 3981-3989, arXiv: gr-qc/9310027.

[6] J. R. Gott III, Phys. Rev. Lett., 66:9 (1991), 1126-1129.

[7] J. Friedman, M. S. Morris, I. D. Novikov, F. Echeverria, G. Klinkhammer, K. S. Thorne, U. Yurtsever, Phys. Rev. D, 42:6 (1990), 1915-1930.

[8] И. Я. Арефьева, И. В. Волович, Т. Ишиватари, ТМФ, 157:3 (2008), 334-344, arXiv: 0903.0567.

[9] J.L. Friedman, "The Cauchy problem on spacetimes that are not globally hyperbolic", The Einstein Equations and the Large Scale Behavior of Gravitational Fields, eds. P. T. Chruściel, H. Friedrich, Birkhäuser, Basel, 2004, 331-346, arXiv: gr-qc/0401004.

[10] J. L. Friedman, M.S. Morris, Comm. Math. Phys., 186:3 (1997), 495-529, arXiv: gr-qc/9411033.

[11] И. В. Волович, О. В. Грошев, Н. А. Гусев, Э. А. Курьянович, "О решениях волнового уравнения на неглобально гиперболическом многообразии", Избранные вопросы математической физики и р-адического анализа, Сб. статей, Тр. МИАН, 265, ред. И. В. Волович, Е. Ф. Мищенко, 2009, 273-287, arXiv: 0903.0741.

[12] A. Chamblin, G. W. Gibbons, A. R. Steif, Phys. Rev. D, 50:4 (1994), R2353-R2355, arXiv: gr-qc/9405001.

[13] U. Yurtsever, Gen. Rel. Grav., 27:6 (1995), 691-693, arXiv: gr-qc/9409040.

[14] I. Ya. Aref'eva, I. V. Volovich, Int. J. Geom. Methods Mod. Phys., 5:4 (2008), 641-651, arXiv: 0710.2696. 